\author{
dspace.vutbr.cz
}

\title{
Transimpedance type MOS-C bandpass analog filter core circuits
}

CEVIK, I.; METIN, B.; HERENCSÁR, N.; CICEKOGLU, 0.; KUNTMAN, H.

Analog Integrated Circuits and Signal Processing

2021, vol. 106, iss. 3, pp. 543-551

ISSN 1573-1979

DOI: http://dx.doi.org/10.1007/s10470-020-01754-2

Accepted manuscript

This is a post-peer-review, pre-copyedit version of an article published in Analog Integrated Circuits and Signal Processing. The final authenticated version is available online at: http://dx.doi.org/10.1007/s10470-020-01754-2 


\title{
Transimpedance type MOS-C bandpass analog filter core circuits
}

\author{
Ismail Cevik $^{1 \bowtie}$, Bilgin Metin ${ }^{2}$, Norbert Herencsar ${ }^{3}$, Oguzhan Cicekoglu $^{4}$, and Hakan Kuntman ${ }^{1}$ \\ ${ }^{1}$ Department of Electronics and Communication Eng., Istanbul Technical University, Maslak, Istanbul, 34469, \\ Turkey \\ E-mail: ceviki@itu.edu.tr, kuntman@itu.edu.tr \\ ${ }^{2}$ Department of Management Information Systems, Bogazici University, Bebek, Istanbul, 34342, Turkey \\ E-mail: bilginx@boun.edu.tr \\ ${ }^{3}$ Department of Telecommunications, Brno University of Technology, Technicka 3082/12, 61600 Brno, Czech \\ Republic \\ E-mail: herencsn@feec.vutbr.cz \\ ${ }^{4}$ Department of Electrical and Electronics Engineering, Bogazici University, Bebek, Istanbul, 34342, Turkey \\ E-mail: cicekogl@boun.edu.tr \\ ${ }^{凶}$ Corresponding author
}

\begin{abstract}
In this paper, we present six area-efficient transimpedance type second-order analog filters. There are many applications where the available signal is current, however the necessary signal for further processing is voltage type. For such applications the presented circuits will be a useful solution. The technique employed is called MOS-only technique and to the best of our knowledge this is the first attempt to implement transimpedance type filters with MOS-only technique. Starting from the core circuit biasing is illustrated and the functionality is shown with LT SPICE simulations using TSMC $0.18 \mathrm{u}$ technology parameters. From six core circuits one circuit is selected and the design is completed for illustration purpose.
\end{abstract}

Keywords MOS Only Filter; MOS-C Filter, Band Pass filter, Transimpedence Filter, Analog Filter

\section{Introduction}

For an analog filter, in general the input and output variables can be selected independently as voltage or current and this permits four types of circuits, namely voltage mode, current mode transimpedance mode, and transadmittance mode filters. The first type was dominating for many decades, it is used in industrial designs and taught in textbooks, the designs are mainly based on opamps, however current mode counterparts received also attention in the last several decades [1-4]. In many publications the latter type employed current conveyors or similar active elements derived from it. For higher frequencies, other types of filters (e.g. [5, 6]), gm-RL [7], or gm-C [8] type filters can be used.
Transimpedance or transadmittance types are also useful depending on application and some related design examples are published before [9-11]

MOS-only approach for circuit design is an alternative approach receiving significant attention during the last decade due to new possibilities it offers [12]. Simplicity in design, component reduction, increase in operating frequency and low cost are some of the advantages. A further advantage is their direct plug-in possibility to the analog or mixed-signal integrated circuit (IC) design. However, this needs some IC design experience if the design needs to be optimized considering other hardware existing in the parent design. MOS-only circuits will roughly fall into two different categories. The first group employs the gate-to-source $\left(C_{\mathrm{gs}}\right)$ parasitic capacitance of the MOS transistor together with its transconductance $g_{\mathrm{m}}$ to obtain the filtering function. Highest possible frequencies with MOS technology can be achieved. There is some concern on about this type of filters. They will suffer from large manufacturing tolerances and nonlinearity of $C_{\mathrm{gs}}$. The tolerance problem can be solved with automatic tuning techniques. The second group employs MOS capacitors or poly-poly capacitors as the filter capacitor to eliminate the mentioned nonlinear behavior. Polypoly capacitors can also be employed in the first group to reduce nonlinearity and to decrease the variation due to the tolerance issue. Previously presented related circuits from MOS-only literature are [12-14]].

Transimpedance type MOS-only circuits (or MOS-C) which fall into the second category were presented in 


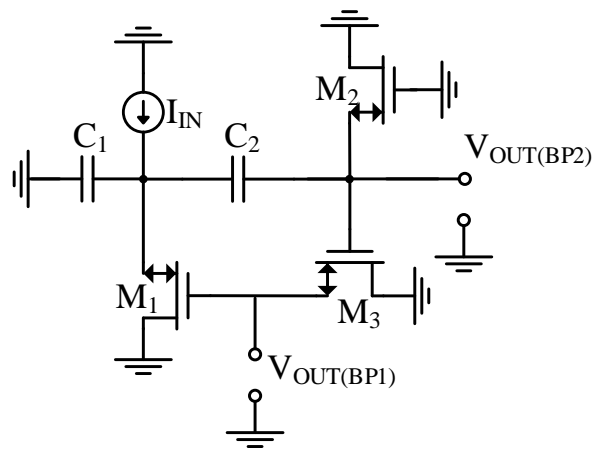

(a)

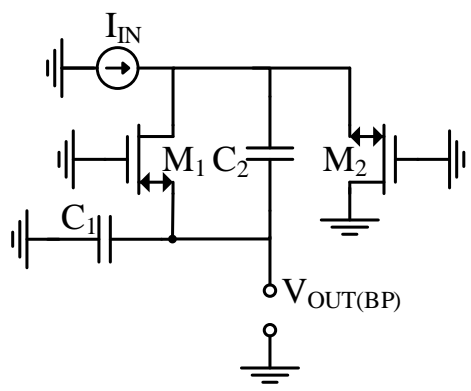

(b)

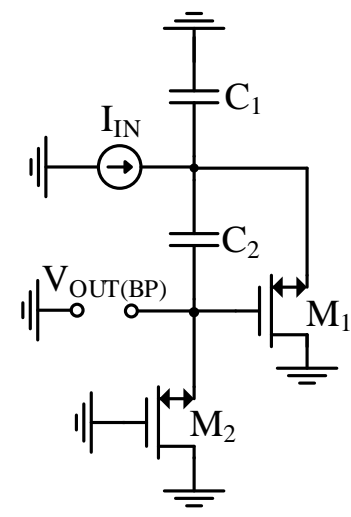

(c)

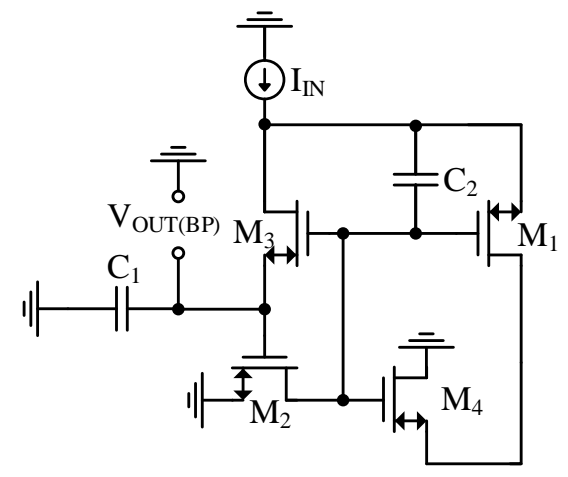

(d)

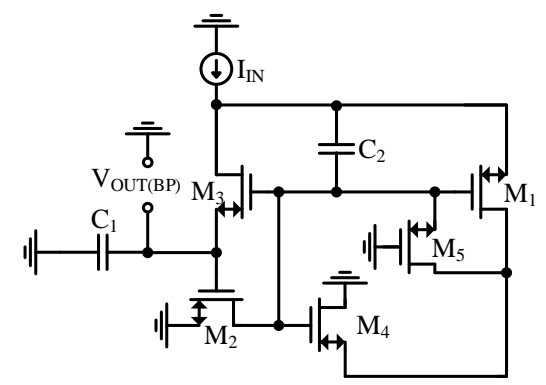

(e)

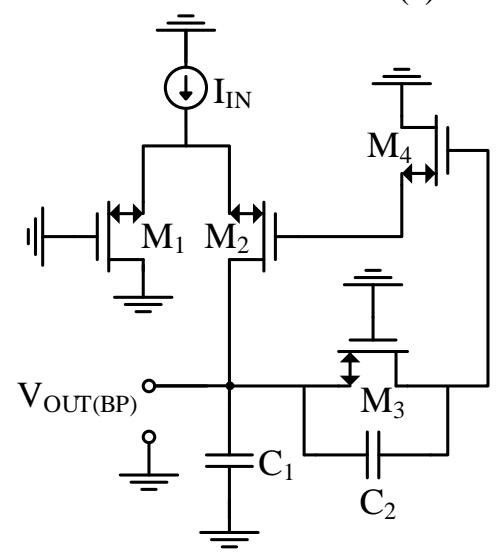

(f)

Fig. 1. The core circuits of the proposed transimpedance filters (without bias)

[14]. In this study we present a total of six transimpedance type MOS-only circuits (or MOS-C) which fall into this category. In many of the publications in the literature active component count is a comparison parameter in analog filter designs. This is due to the fact that reduced chip area is expected from these filters and also one expects lower power consumption [12]. The general philosophy of MOSFET-only design approach is as follows: In these circuits only metal-oxidesemiconductor transistors operating in the saturation region are employed instead of generic active building blocks. Therefore instead of a passive resistive element, the transconductance, $g_{\mathrm{m}}$ parameter of MOS transistor is used to obtain resistive (or conductive) dimension. The presented circuits in this study, are easily integrable to an existing analog design with some integrated circuit design experience. They can also be used as independent filter circuits. The design procedure is shown to give guidelines to the IC design engineer.

\section{The Six Proposed Transimpedance Filter Circuits}

Transimpedance type filters cannot be cascaded with identical filters due to the fact that the input signal is a current and output signal is a voltage. However, these filters are suitable to be used at appropriate interfaces and intermediate stages where the signal types are in desired form.

The six proposed transimpedance type MOS-Only analog filter circuits without biasing components are shown in Fig. 1. These circuits are the core circuits and need DC bias to operate the transistors in saturation regime. The double-sided arrows mean each transistor can be selected as either NMOS or PMOS transistors. However once they are selected the biasing circuit should be designed appropriately. In fact they represent parent circuits and choosing different combinations may result different versions. On the other hand not all of 
them may permit biasing in such cases additional transistors may be needed.

When all secondary effects and parasitics of transistors are ignored and they are modeled as ideal transconductors, transfer functions for the first three of these circuits (Fig.1a,b,c) are identical and given in Eq. 1.

$$
H(s)=\frac{s C_{2}}{s^{2} C_{1} C_{2}+s\left(C_{1}+C_{2}\right) g_{m 2}+g_{m 1} g_{m 2}}
$$

Resonant angular frequency and quality factor for these three circuits are given in Eq. 2.

$$
\omega_{0}=\sqrt{\frac{g_{m 1} g_{m 2}}{C_{1} C_{2}}} \quad Q=\frac{1}{\left(C_{1}+C_{2}\right)} \sqrt{\frac{g_{m 1} C_{1} C_{2}}{g_{m 2}}}
$$

Note that the circuits in Fig. 1a and Fig.1c are related and $g_{m 3}$ in Fig. 1a does not appear in the transfer function. Since transistor M3 in Fig. 1a acts as a perfect voltage buffer when all transistors are ideal transconductors, signals at the two nodes labeled as $\mathrm{V}_{\text {OUT(BP1) }}$ and $\mathrm{V}_{\text {OUT(BP2) }}$ are identical. Comparison of both circuits illustrates how additional transistors can be inserted without changing the transfer function. This fact is useful when considering biasing conditions. Here M3 does not change the ac analysis results but acts as a dc voltage level shifter. Inserting such transistors appropriately permits easy biasing whenever necessary. Moreover if these cores are used as a plug-in circuit these techniques will be useful when interfacing circuit blocks with different dc levels.

Transfer function for the fourth circuit shown in Fig. 1d is given in Eq. 3 .

$$
H(s)=\frac{s C_{2}}{s^{2} C_{1} C_{2}+s C_{2} g_{m 2}+g_{m 1} g_{m 2}}
$$

Resonant angular frequency and quality factor for this circuit is given in Eq. 4.

$$
\omega_{0}=\sqrt{\frac{g_{m 1} g_{m 2}}{C_{1} C_{2}}} \quad Q=\sqrt{\frac{g_{m 1} C_{1}}{g_{m 2} C_{2}}}
$$

Transfer function for the fifth circuit shown in Fig. 1e is given in Eq. 5 .

$$
H(s)=\frac{s}{C_{1}\left(1+\frac{g_{m 5}}{g_{m 3}}\right)\left(s^{2}+s \frac{\omega_{0}}{Q}+\omega_{0}^{2}\right)}
$$

Resonant angular frequency and quality factor for this circuit is given in Eq. 6.

$$
\omega_{0}=\sqrt{\frac{g_{m 1} g_{m 3}\left(g_{m 2}+g_{m 5}\right)}{C_{1} C_{2}\left(g_{m 3}+g_{m 5}\right)}}
$$

$$
Q=\frac{\sqrt{g_{m 1} g_{m 3}\left(g_{m 2}+g_{m 5}\right) C_{1} C_{2}\left(g_{m 3}+g_{m 5}\right)}}{\left(C_{1} g_{m 1} g_{m 5}+C_{2}\left(g_{m 2} g_{m 3}+g_{m 5} g_{m 3}\right)\right)}
$$

Transfer function for the sixth circuit shown in Fig. 1f is given in Eq. 7.

$$
H(s)=\frac{s}{C_{1}\left(1+\frac{g_{m 1}}{g_{m 2}}\right)\left(s^{2}+s \frac{\omega_{0}}{Q}+\omega_{0}^{2}\right)}
$$

Resonant angular frequency and quality factor for this circuit is given in Eq. 8.

$$
\omega_{0}=\sqrt{\frac{g_{m 1} g_{m 2} g_{m 3}}{C_{1} C_{2}\left(g_{m 1}+g_{m 2}\right)}} \quad Q=\sqrt{\frac{g_{m 3} C_{1}\left(g_{m 1}+g_{m 2}\right)}{C_{2} g_{m 1} g_{m 2}}}
$$

Note that here $g_{m 4}$ does not appear in the transfer function thus it does not effect the ac response. However it shifts the dc level. The transfer function is similarly independent from $g_{m 4}$ for the circuit in Fig. 1e. Since ideal ac models of both NMOS and PMOS transistors are identical they can be selected arbitrarily as long as biasing is possible.

\section{Circuit Design Process with single spacing}

The actual design starts by picking up a core circuit, selecting transistor types NMOS or PMOS and biasing it properly. Filter circuits in Fig. 1a and Fig. 1b were studied in detail in [13]. We selected the circuit in Fig. $1 \mathrm{~d}$ in this study for illustration purpose. Complete circuit with bias is shown in Fig. 2. Since there is no high impedance node in the circuit, biasing circuit is relatively simple. Transistors M8-M11 in Fig. 2 are the current mirrors biasing the core transistors. Transistors M5-M7 are used to bias these current mirrors.

Selected filter circuit is designed for a center frequency of $7 \mathrm{MRad} / \mathrm{s}$ which corresponds $1.13 \mathrm{MHz}$. $\mathrm{C} 1$ and $\mathrm{C} 2$ capacitor values are $10 \mathrm{pF}$ and $1 \mathrm{pF}$ respectively. $10 \mathrm{pF}$ capacitors can be built in a compact area with Double Metal-Insulator-Metal (Double MIM) capacitors in standard CMOS processes. The circuit is

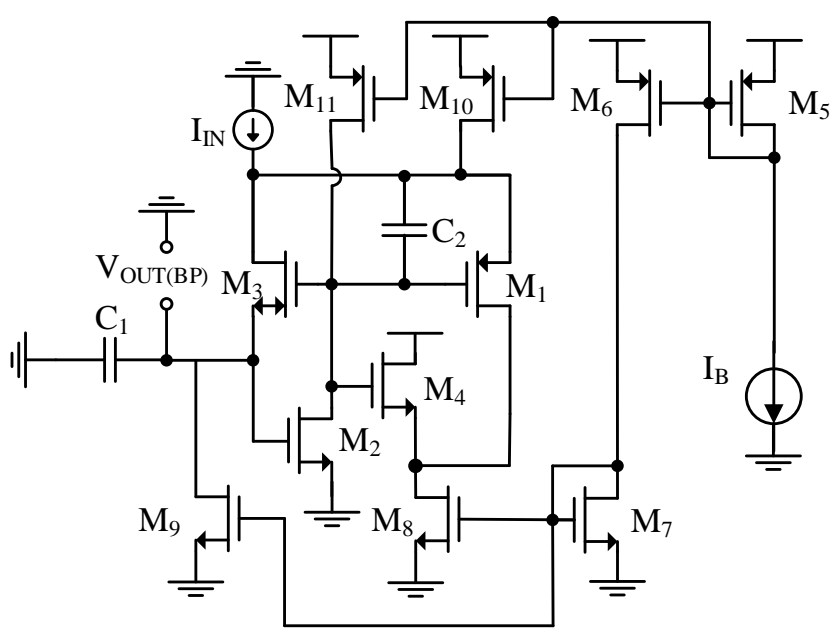

Fig. 2. $4^{\text {th }}$ transimpedance filters with biasing 
Table 1. Device sizes and bias currents

\begin{tabular}{|l|c|c|c|c|c|c|c|c|c|}
\hline & M1 & M2-M4 & M5-M7 & M8 & M9 & M10 & M11 & C1 & C2 \\
\hline $\mathbf{W}(\boldsymbol{\mu m})$ & 5 & 2 & 1 & 5 & 10 & 10 & 5 & & \\
\hline $\mathbf{L}(\boldsymbol{\mu m})$ & 0.5 & 0.5 & 1 & 1 & 1 & 1 & 1 & & \\
\hline $\mathbf{I}(\boldsymbol{\mu} \mathbf{A})$ & 1 & 1 & 0.2 & 1 & 2 & 2 & 1 & & \\
\hline Cap (pF) & & & & & & & & 10 & 1 \\
\hline
\end{tabular}

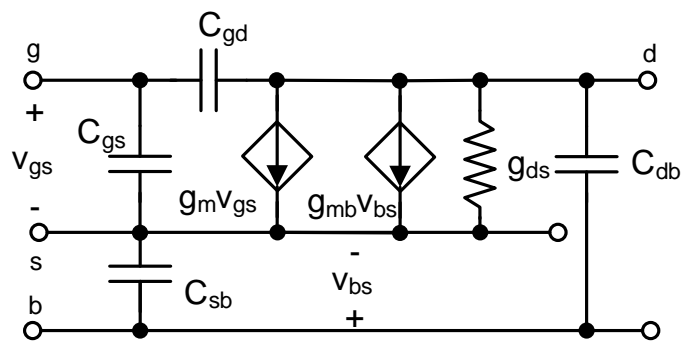

Fig. 3. High frequency small signal model of MOSFET

realized in a $180 \mathrm{~nm}$ CMOS process. Lengths of all core transistors are chosen as $500 \mathrm{~nm}$ to reduce channel length modulation and other short channel effects. Widths of NMOS transistors are chosen as $2 \mu \mathrm{m}$ and widths of PMOS transistors are chosen as $5 \mu \mathrm{m}$. Transistor transconductances are approximately $20 \mu \mathrm{S}$ for these sizes for a bias current of $1 \mu \mathrm{A}$.

However, a transistor is not a perfect transconductor. Channel Length Modulation acts as a small signal resistor between drain and source $\left(g_{\mathrm{ds}}\right)$ and Body Effect acts as a second transconductor controlled by bulk to source voltage $\left(g_{\mathrm{mb}}\right)$. A more complete small signal transistor model including these effects and parasitic capacitances is shown in Fig. 3. Even this model is incomplete and does not reflect short channel effects such as Gate Induced Drain Leakage (GIDL), Drain induced Barrier Lowering (DIBL), Substrate Current Induced body effect (SCBE). These short channel effects have significant effects on transistor behavior in submicron CMOS processes. When these filter circuits are implemented with real transistors, their behavior deviates from the ideal transconductor implementation.

The most problematic nonideal effect is the channel conductance $g_{\mathrm{ds}}$ created by channel length modulation. It creates a parasitic signal path between drain and source of the transistor. When drain of a transistor is on the signal path, this component will create significant deviation. When sources or drains of all transistors in a filter topology are grounded, backgate conductance transconductance $g_{\mathrm{mb}}$ and channel conductance $g_{\mathrm{ds}}$ have no effect or act as resistors from source or drain to ground. However, if both drain and source of a transistor is on the signal path, the feed-forward path through the channel conductance of the transistor will create a zero. Any filter topology with a common gate transistor will suffer from this problem. The added zero will change filter response. Source follower or common source amplifier based filter topologies will act closer to the ideal response.

Channel conductances of M2 and M3 transistors in the $4^{\text {th }}$ filter circuit shown in Fig. 1d provide additional signal paths. Channel conductance of M3 provide a direct connection from input node to output node. Channel conductance of $\mathrm{M} 2$ connect $\mathrm{C} 2$ to ground. These parallel signal paths move the filter zero to a non zero value.

The transfer function for the fourth filter circuit including the channel conductances of M2 and M3 parasitic capacitors is given in Eq. 9. This equations are simplified considering the fact that channel conductances are much smaller than transconductances for saturated transistors.

$$
H(s)=\frac{s C_{2} g_{m 3}+g_{d s 2} g_{d s 3}}{g_{m 3} C_{1} C_{2} s^{2}+g_{m 2} g_{m 3} C_{2} s+g_{m 1} g_{d s 2} C_{1} s+g_{m 1} g_{m 2} g_{m 3}}
$$

This equation clearly shows that zero is not at zero frequency. The channel resistances do not change the second order nature of the transfer function as shown by the denominator. Center frequency, zero frequency and quality factor in this non ideal case are shown in Eq. 10.

$$
\begin{gathered}
\omega_{0}=\sqrt{\frac{g_{m 1} g_{m 2}}{C_{1} C_{2}}} \omega_{z}=\frac{g_{d s 2} g_{d s 3}}{C_{2} g_{m 3}} \\
Q=\frac{g_{m 3} \sqrt{g_{m 1} g_{m 2} C_{1} C_{2}}}{g_{m 2} g_{m 3} C_{2}+g_{m 1} g_{d s 2} C_{1}}
\end{gathered}
$$

Parasitic capacitances will also affect the frequency response. However, filter capacitors are chosen much larger than transistor parasitic capacitances. Thus their effects will not be significant. Gate to drain $\left(\mathrm{C}_{\mathrm{gd}}\right)$ and gate to source $\left(\mathrm{C}_{\mathrm{gs}}\right)$ parasitic capacitances provide signal paths in parallel with transistors while drain to bulk $\left(\mathrm{C}_{\mathrm{db}}\right)$ and source to bulk $\left(\mathrm{C}_{\mathrm{sb}}\right)$ depletion capacitances provide a path from circuit nodes to ground. Effect of $\mathrm{C}_{\mathrm{db}}$ and $\mathrm{C}_{\mathrm{sb}}$ is shorting circuit nodes to ground at very high frequency, so they introduce additional poles at very high frequencies. However, the parallel signal paths introduced by $\mathrm{C}_{\mathrm{gd}}$ and $\mathrm{C}_{\mathrm{gs}}$ introduce both zeros and poles.

Capacitors in the circuit can be grouped into four capacitors from circuit nodes to ground and 3 capacitors between circuit nodes. These capacitors are given in Eq. 11. 


$$
\begin{gathered}
C_{n 1}=C_{s b 1}+C_{d b 3} \quad C_{n 2}=C_{g d 4}+C_{d b 2} \\
C_{n 3}=C_{d b 1}+C_{s b 4} \\
C_{1}+C_{g s 2}+C_{s b 3} \\
C_{n 12}=C_{2}+C_{g d 3}+C_{g s 1} \quad C_{n 23}=C_{g d 1}+C_{g s 4} \\
C_{n 24}=C_{g d 2}+C_{g s 3}
\end{gathered}
$$

Since $\mathrm{C}_{\mathrm{gs} 1}, \mathrm{C}_{\mathrm{gs} 2}$ and are in parallel with main filter capacitances, their effect is simply increasing filter capacitor values. Even if transistors M1 and M2 are relatively large and $\mathrm{C}_{\mathrm{gs} 1}$ and $\mathrm{C}_{\mathrm{gs} 2}$ are not negligible compared to filter capacitors, these added parasitic capacitors will not affect the filter response. However, we must keep in mind that increasing transistor sizes will also increase depletion capacitors $\mathrm{C}_{\mathrm{db}}$ and $\mathrm{C}_{\mathrm{sb}}$, ie. $\mathrm{C}_{\mathrm{n} 1}, \mathrm{C}_{\mathrm{n} 2}$ and $\mathrm{C}_{\mathrm{n} 3}$ will increase. When these capacitors increase, the high frequency poles introduced by these capacitors will move to lower frequencies.

The transfer function for the fourth filter circuit when all parasitic capacitances are included is a very complicated function with 3 zeros and 4 poles. Transfer function can be simplified significantly when $C_{1}$ and $C_{2}$ are assumed to be much larger than all parasitic capacitances. Since all transistors are operating in saturation, gate to drain capacitors are much smaller than other parasitic capacitances. Therefore, equations can be further simplified ignoring $\mathrm{C}_{\mathrm{gd}}$ capacitors in comparison to other capacitors. $\mathrm{C}_{\mathrm{n} 4}$ and $\mathrm{C}_{\mathrm{n} 12}$ are larger than all other capacitors. This equation can be simplified to the form including the parasitic capacitors is given in Eq. 12.

$H(s) \cong \frac{s C_{n 12}\left(g_{m 3}+s C_{n 24}\right)\left(g_{m 4}+s\left(C_{n 3}+C_{n 23}\right)\right)}{a_{4} s^{4}+a_{3} s^{3}+a_{2} s^{2}+C_{n 12} g_{m 2} g_{m 3} g_{m 4} s+g_{m 1} g_{m 2} g_{m 3} g_{m 4}}$

where

$$
\begin{gathered}
a_{4}=C_{n 12} C_{n 4}\left(C_{n 23}\left(2 C_{n 1}+2 C_{n 2}+C_{n 3}\right)\right. \\
\left.+C_{n 24}\left(C_{n 3}+C_{n 23}\right)\right) \\
a_{3}=C_{n 12} C_{n 4}( \\
\left(C_{n 3}+C_{n 23}\right) g_{m 3} \\
\left.+\left(C_{n 1}+C_{n 2}+C_{n 24}\right) g_{m 4}\right)
\end{gathered}
$$

$a_{2}=C_{n 12} C_{n 4} g_{m 3} g_{m 4}$

Resonant angular frequency and quality factor for this circuit are given in Eq. 13. As long as parasitic capacitances are much smaller than filter capacitances, center frequency and quality factor simplifiers to the values given in Eq. 4.

$$
\begin{gathered}
\omega_{0} \cong \sqrt{\frac{g_{m 1} g_{m 2}}{C_{n 4} C_{n 12}} \cong \sqrt{\frac{g_{m 1} g_{m 2}}{C_{1} C_{2}}}} \\
Q \cong \sqrt{\frac{C_{n 4} g_{m 1}}{C_{n 12} g_{m 2}}} \cong \sqrt{\frac{C_{1} g_{m 1}}{C_{2} g_{m 2}}}
\end{gathered}
$$

Eq. 12 shows that there are two extra zeros in addition to the filter zero at $0 \mathrm{~Hz}$ and 2 additional poles. These extra zeros and poles affect filter response. When the filter is designed for high $\mathrm{Q}$, first two poles will be complex. $3^{\text {rd }}$ and $4^{\text {th }}$ poles will be real. Approximate values of the extra poles and zeros are given in Eq.14.

$$
\begin{gathered}
\omega_{z 1} \cong g_{m 4} /\left(C_{n 3}+C_{n 23}\right) \quad \omega_{z 2}=g_{m 3} / C_{n 24} \\
\omega_{p 3} \cong\left(\alpha+\frac{1}{\omega_{z 1}}+\frac{1}{\omega_{z 3}}\right)^{-1} \\
\omega_{p 4} \cong\left(\frac{1}{\omega_{z 2}}+\beta\right)^{-1}+\left(\frac{\gamma}{\omega_{z 1}}+\delta\right)^{-1}
\end{gathered}
$$

where

$$
\begin{array}{ll}
\alpha=\frac{C_{n 1}+C_{n 2}}{g_{m 3}} & \beta=\frac{C_{n 23}\left(2 C_{n 1}+2 C_{n 2}+C_{n 3}\right)}{g_{m 3}\left(C_{n 3}+C_{n 23}\right)} \\
\gamma=\frac{C_{n 24}}{C_{n 1}+C_{n 2}+C_{n 24}} & \delta=\frac{C_{n 23}\left(2 C_{n 1}+2 C_{n 2}+C_{n 3}\right)}{\left(C_{n 1}+C_{n 2}+C_{n 24}\right) g_{m 4}}
\end{array}
$$

From Eq. 14 one can conclude that the extra poles and zeros are created by transistor parasitics. On the other hand $1^{\text {st }}$ and $2^{\text {nd }}$ poles are linked to $\omega_{0}$ and $\mathrm{Q}$. Therefore, first 2 poles are determined by filter capacitances $C_{1}$ and $\mathrm{C}_{2}$. If parasitic capacitances are negligible compared to main $\mathrm{C}_{1}$ and $\mathrm{C}_{2}$, these extra zeros and poles will be at relatively high frequencies compared to the first 2 poles.

Even the simplified pole and zero expressions in Eq. 15 depend on too many parameters. In order to see the sequence of these values in typical cases, we will calculate these frequencies for device sizes and currents in Table 1. We assume source and drain regions have same geometry and ignore voltage dependence of depletion capacitances. Since size and current of M3 and $\mathrm{M} 4$ are equal, transconductances $\mathrm{g}_{\mathrm{m} 3}$ and $\mathrm{g}_{\mathrm{m} 4}$ will be equal. Since channel size and length of source and drain regions are equal, bulk capacitors and gate to source capacitors of NMOS transistors M2-M4 are equal. Gate to source capacitance of M1 will be 2.5 times those of NMOS transistors. Depletion capacitance will be approximately 2.5 times if we assume length of source and drain regions also increase 2.5 times. Gate to drain 
capacitors can be ignored since they are much smaller than gate to source capacitors. Zero and pole positions under these assumptions are given in Eq. $15 . \mathrm{C}_{\mathrm{b}}$ is either $\mathrm{C}_{\mathrm{db}}$ or $\mathrm{C}_{\mathrm{sb}}$ of and NMOS transistor and Cgs is gate to source capacitance of NMOS transistors.

$$
\begin{gathered}
\omega_{z 1} \cong 2 g_{m} /\left(2 C_{g s}+7 C_{b}\right) \quad \omega_{z 2} \cong g_{m} / C_{g s} \\
\omega_{p 3} \cong g_{m} /\left(2 C_{g s}+8 C_{b}\right) \\
\omega_{p 4} \cong g_{m} / 2 C_{g s}+3 g_{m} /\left(2 C_{g s}+32 C_{b}\right)
\end{gathered}
$$

$\omega_{\mathrm{p} 3}$ is the smallest one. $\omega_{\mathrm{z} 1}$ is approximately $2 \omega_{\mathrm{p} 3} . \omega_{\mathrm{p} 4}$ is larger than $\omega_{\mathrm{z} 1} . \omega_{\mathrm{z} 2}$ is larger than $\omega_{\mathrm{p} 4}$ unless $\mathrm{C}_{\mathrm{gs}}$ is very large compared to $\mathrm{C}_{\mathrm{d}}$. Slope of filter response will increase after each pole and decrease after each zero. It will go back to its ideal value at frequencies larger than $\omega_{\mathrm{z} 2}$ since they neutralize each other. Actual response and ideal response will be parallel at high frequencies, but magnitude of actual response will be smaller than ideal response due to faster role off between $3^{\text {rd }}$ pole and $2^{\text {nd }}$ zero. Eq. 15 is an approximation. Actual pole and zero values will be different, but it is useful for understanding how frequency response behaves.

\section{Simulation Results}

Bandpass filter circuit is implemented with ideal voltage controlled current sources and real transistors in $180 \mathrm{~nm}$ TSMC CMOS process in order to compare ideal circuit behavior and simulated behavior. All branches in the circuit are biased with $1 \mu \mathrm{A}$ current. Core filter consumes $7.36 \mu \mathrm{W}$. Biasing branches are sized smaller than core circuit branches in order to minimize power consumption. Filter biasing branches consume $418 \mathrm{nW}$.

Ideal and simulated frequency responses of the first filter are shown in Fig. 4. Center frequency of ideal response is at $1.1 \mathrm{MHz}$ as anticipated from calculations. Center frequency of simulated response increases to $1.13 \mathrm{MHz}$ and transimpedance gain decreases with real transistors as expected. Peak value of the transfer function decreases $3.5 \mathrm{~dB}$ compared to ideal response. However, bandpass characteristics are preserved with real transistors. Parasitic capacitances do not have a significant effect because the parasitics associated with the relatively small transistors are negligible compared to $C_{1}$ and $C_{2}$.

The biggest deviation from ideal behavior is the flat low-frequency response. Circuits act as bandpass filter for a wide range of frequencies, but magnitude roll-off stops at frequencies lower than the zero created by the channel conductances of transistor M2 and M3. However, there is $90 \mathrm{~dB}$ roll-off from the peak value and effects of this zero are negligible. Channel conductances

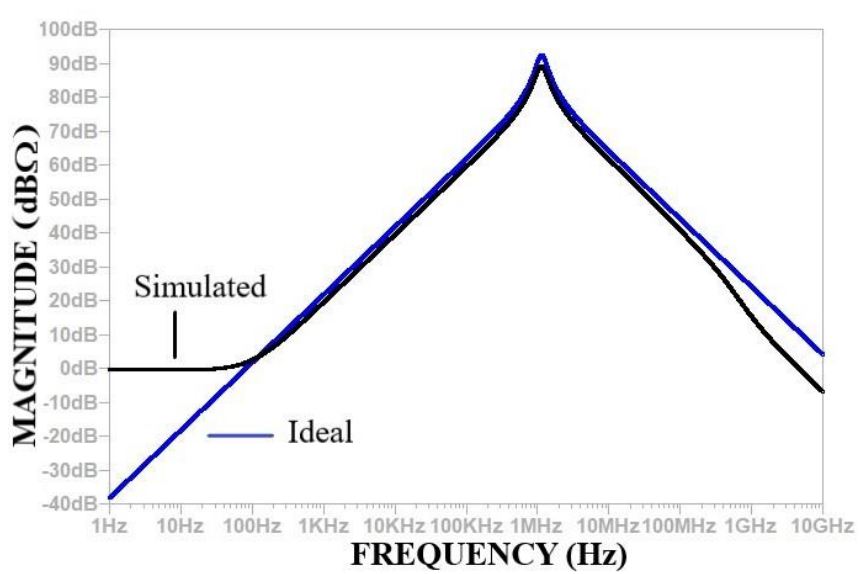

Fig. 4. Frequency Response of the $4^{\text {th }}$ TI Filter

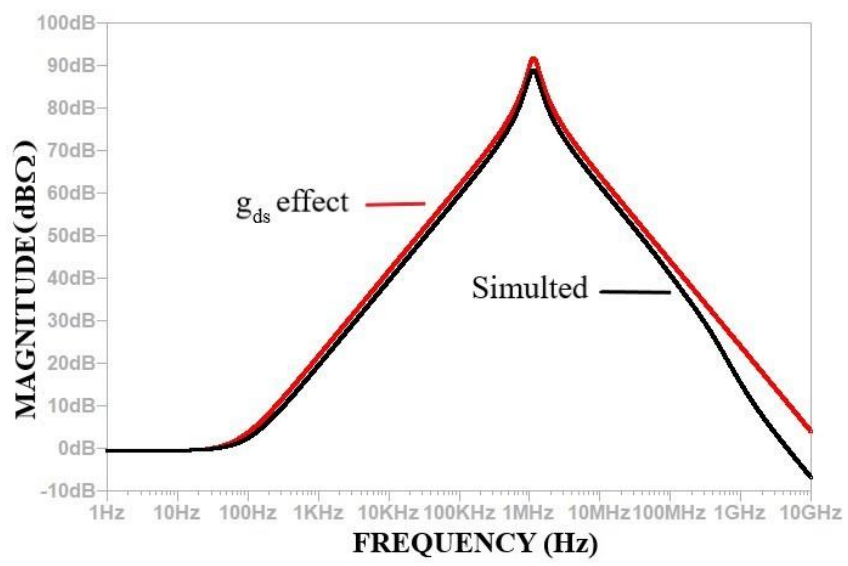

Fig. 5. Frequency Response of the ideal model of $4^{\text {th }}$ TI Filter with channel conductances

of M2 and M3 are added the ideal model to verify the source of low frequency flatting. Fig. 5 shows comparison of this semi ideal model and the actual simulated response. This result clearly shows that the low frequency flatting is the result of channel conductances of M3 and M4. The zero frequency of the model and the actual circuit overlap perfectly.

Parasitic capacitances are responsible for deviations from the ideal behavior at high frequencies as anticipated in Section 3. Fig. 4 shows that simulated response rolls off faster than the expected response. Slope of the simulated response is not constant in the frequency range from $250 \mathrm{MHz}$ to $1.5 \mathrm{GHz}$. After this frequency range, simulated curve and ideal curve become parallel. This behavior can be explained by 2 poles and 2 zeros positioned in a pole, zero, pole, zero sequence. This exactly what was anticipated from Eq. 15. Simulated response follows the ideal response for more than 2 decades. Since Eq. 15 is just a This means frequency drops more than $40 \mathrm{~dB}$ before deviations become significant. Moreover, a faster roll off at out of band frequencies is not an undesired property in a filter.

Simulated center frequency shows a good match with ideal response. In fact all analog filters are subject to automatic on chip tuning. Slight deviations in center frequency can easily be compensated. 


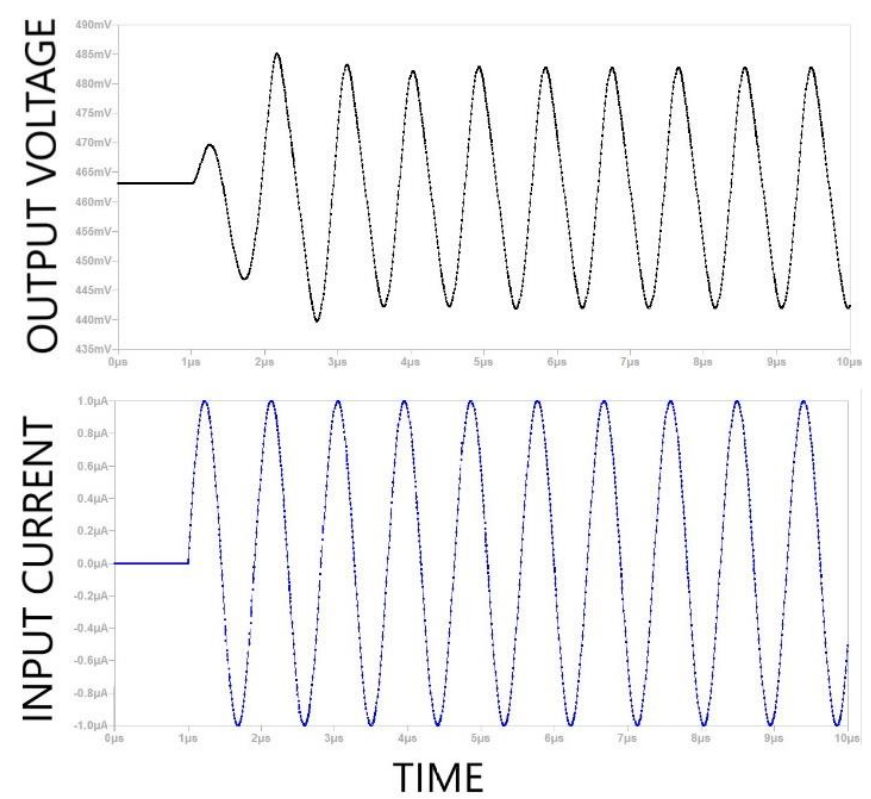

Fig. 6. Time-domain response of $4^{\text {th }}$ TI filter circuit

The time-domain response of the filter circuit to sinusoidal input current is shown Fig. 6. Input current to the circuit is at the center frequency of the circuit. The amplitude of the input current is $1 \mu \mathrm{A}$. The filter response settles within two periods after the application of the input signal. Input current and output voltage are in phase as expected since the phase shift is $0^{\circ}$ at the center frequency.

\section{Conclusions}

In this study we presented six core circuits operating as transimpedance filter and are of MOS-C (MOS-only) type. It is shown with ideal and nonideal analyses that these circuits perform as very good filter circuits. To illustrate the functionality and the design procedure one of the selected core circuits is biased properly and simulated with LT SPICE using TSMC 0.18u technology parameters. The presented circuits will be useful in applications where the available signal is current but the signal processing is performed in voltage mode.

\section{References}

[1] Toumazou, C., Lidgey, F. J., \& Haigh, D. G. (Eds.). (1993). Analogue IC Design: The Current-Mode Approach. Institution of Engineering and Technology.

[2] Gungordu, A. D., Altun, M., \& Cevik, I. (2017). Low input resistance current buffer stage using a controllable positive feedback loop, and applications of current conveyor based filters. AEU - International Journal of Electronics and Communications, 82, 58-65. https://doi.org/10.1016/j.aeue.2017.07.034

[3] Kumngern, M., \& Khateb, F. (2019). Current-mode universal filter and quadrature oscillator using current controlled current follower transconductance amplifiers. Analog Integrated Circuits and Signal Processing, 100(2), 235-248. https://doi.org/10.1007/s10470-0181345-8

[4] Horng, J.-W. (2019). High Output Impedance Current-Mode Universal First-Order Filter With Three Inputs Using One DDCC. Recent Advances in Electrical \& Electronic Engineering, 3(12), 242-246. https://doi.org/10.2174/2352096511666180525124701

[5] Herencsar, N., Minaei, S., Koton, J., Yuce, E., \& Vrba, K. (2013). New resistorless and electronically tunable realization of dual-output VM allpass filter using VDIBA. Analog Integrated Circuits and Signal Processing, 74(1), 141-154. https://doi.org/10.1007/s10470-012-99362

[6] Abaci, A., \& Yuce, E. (2018). Voltage-mode first-order universal filter realizations based on subtractors. AEU - International Journal of Electronics and Communications, 90, 140-146. https://doi.org/10.1016/j.aeue.2018.04.017

[7] Ahmadi, P., Taghavi, M. H., Belostotski, L., \& Madanayake, A. (2015). A $0.13-\mu \mathrm{m}$ CMOS Current-Mode All-Pass Filter for Multi-GHz Operation. IEEE Transactions on Very Large Scale Integration (VLSI) Systems, 23(12), 2813-2818. https://doi.org/10.1109/TVLSI.2014.2380820

[8] Ozenli, D., Yesil, A., Arslan, E., \& Kuntman, H. H. (2018). Novel First Order Current Mode MOS-C Phase Shifters. Elektronika ir Elektrotechnika, 24(1), 31-35. https://doi.org/10.5755/j01.eie.24.1.20157

[9] Tan, L., Li, Q., Li, R., \& Teng, J. (2013). Design of transimpedance low-pass filters. International Journal of Electronics, 100(2), 142-149. https://doi.org/10.1080/00207217.2012.680182

[10] Beg, P., Maheshwari, S., \& Siddiqi, M. A. (2013). Digitally controlled fully differential voltage- and transadmittance-mode biquadratic filter. IET Circuits, Devices \& Systems, 7(4), 193-203. https://doi.org/10.1049/iet-cds.2012.0244

[11] Shah, N. A., Quadri, M., \& Iqbal, S. Z. (2007). CDTA based universal transadmittance filter. Analog Integrated Circuits and Signal Processing, 52(1), 65-69. https://doi.org/10.1007/s10470-007-9091-3

[12] Yildiz, H. A., Ozoguz, S., Toker, A., \& Cicekoglu, O. (2013). On the Realization of MOS-Only Allpass Filters. Circuits, Systems, and Signal Processing, 32(3), 1455-1465. https://doi.org/10.1007/s00034-0129500-4

[13] Arslan, E., Metin, B., \& Cicekoglu, O. (2015). MOSFET-only multifunction biquad filter. AEU - International Journal of Electronics and $\begin{array}{lr}\text { Communications, } & 69(12), \\ \end{array}$ https://doi.org/10.1016/j.aeue.2015.07.018

[14] Cevik, I., Metin, B., Herencsar, N., Cicekoglu, O., \& Kuntman, H. (2019). Transimpedance Type MOS-C Bandpass Filter Cores. In 2019 11th International Conference on Electrical and Electronics Engineering (ELECO) (pp. 371-374). Presented at the 2019 11th International Conference on Electrical and Electronics Engineering (ELECO). https://doi.org/10.23919/ELECO47770.2019.8990543 